\title{
TopiGluk Nutritive Mix in the Function of Glucose Homeostasis
}

\author{
Draško Gostiljac* \\ Faculty of medicine, University of Belgrade, Serbia
}

Submission: April 28, 2021; Published: May 11, 2021

*Corresponding author: Draško Gostiljac, Faculty of medicine, University of Belgrade, 11000 Belgrade, Dr Subotica, Serbia

Keywords: Glucose homeostasis; Food stuff; TopiGluk nutritive mix; Diabetes prevention

\section{Biologically Active Substances in Glucose Homeostasis Functioning}

Some trials showed that positive effect on glycoregulation was achieved by enriching food with various biologically active substances of herbal origin. One of the basic food stuff that is usually consumed almost with every meal, is bread. Recommendations so far refer to consuming rye and integral bread with bigger dietary fibers content instead of white bread.

TopiGluk nutritive mix that can be used as a supplement to the mixture for a special bread type made of integral, oat and buckwheat flour, and whose biggest significance is in the effect it has on glucose homeostasis, was formulated by careful selection of biologically active substances of herbal origin. While choosing biologically active substances for TopiGluk formulating, one took care of numerous factors; particularly important are availability of the substance, its impact on glycoregulation, other favorable metabolic effects, its applicability in technological process of bread preparation and manufacturing, as well as impact of certain phases of technological procedure, for example, bread baking temperature impact on active principles of the ingredients (duration), specific smell and taste in planned concentrations, mutual compatibility and cumulative effect on organoleptic properties of the mix, as well as expected effect of TopiGluk on the basis mixture for a special bread type.

\section{TopiGluk and Diabetes Prevention}

TopiGluk is a nutritive mix that is added to the mixture for a special bread type, aimed at qualitative improvement of product, firstly from the aspect of glucose homeostasis. TopiGluk mainly contains fructose polysaccharide due to which fructose gets released instead of glucose during metabolic processes and comparing to the flour that contains mainly starch as the glucose polysaccharide, it affects positively glucose postprandial level and glycoregulation.

The most Significant Biological Active Principles in

\section{TopiGluk Composition}

Basil (Ocimum basilicum) is known by the fact that it has strong antioxidant properties. One of the basil antioxidants is betacarotene. Beta-carotene, which turns into vitamin A in the body, prevents oxidation of cholesterol in circulation system and in that way it protects the heart and blood vessels. Basil is a good source of vitamin B6 and magnesium. Vitamin B6 prevents accumulation of potentially dangerous molecule homocysteine. Magnesium stimulates cardiovascular system wellbeing, by helping relaxation of mussels and blood vessels. One research showed that basil can lower sugar level after meal, which reduces the need for therapy. Ocimum basilicum extract has antidiabetic effects that are probably intermediated by limiting glucose absorption through carbohydrates metabolism inhibition and impact on glucose mobilization from liver. Chronic oral use does not predispose risk of hepatotoxic activities in short period of time. Supplemental researches are related with evaluation of effects on long-term basil extract application in treating diabetes [1].

Garlic (Allium sativum) was safely used since ancient times both in nutrition as well as a cure with human population, but studies about its efficiency in treating diabetes, gave different results. There is a study that evaluated potential hypoglycemic garlic effects in type 2 diabetes. Combination of garlic with typical anti-diabetic medications resulted in supplemental improvement of glycaemia control, beside the antihyperlipidemic effect. It was concluded that garlic can be a good supplement at patients with diabetes and hyperlipidemia [2]. 
Greek seed (Trigonella foenum graecum) is a plant that is often used in cooking, and in traditional medicine in Asia, as a cure for diabetes. It showed that it acutely reduces postprandial glucose level, but the long-term effect on glycoregulation is still insufficiently tested. A survey of the clinical trials of fenugreek effect on glucose homeostasis, which have been carried out so far, shows that results of the respective trials are in favor of favorable fenugreek seed effect on metabolic control at persons with diabetes. However, it is necessary to apply more quality methodology by using a sufficient dose of well-prepared fenugreek preparation in order to obtain more evidence of the respective [3]

Ginger (Zingiber officinale) is safe for use, cost friendly and, based on some clinical trials, it is effective with type 2 diabetes. Zingiber officinale started to be used in therapy purposes as a nutrition supplement due to its extensive therapy potential regarding type 2 diabetes and in preventing diabetes complications by direct interaction with various etiopathogenetic causes of type 2 diabetes. Effects of Zingiber officinale on type 2 diabetes were described, as well as diabetes complications on molecular level. Explanation for anti-diabetic effects of Zingiber officinale includes inhibition of several transcriptional routes, lipid peroxidation, enzymes that regulate carbohydrates metabolism and HMG-CoA reductase and activation of antioxidant protection enzymes and receptors of low-density lipoproteins. So, by aiming those routes, Zingiber officinale manifests its anti-diabetic therapy effect by increasing insulin sensitivity and synthesis, by preservation of beta-cells of pancreatic islets, by lowering fat accumulation, by lowering oxidative stress and by increasing absorption of glycose in tissues. Beside the afore mentioned effects, Zingiber officinale also manifests protective effects for diabetes complication, particularly nephropathy and diabetic cataract, by acting as antioxidant and antiglycemic agent. Zingiber officinale can help in treating T2DM and diabetes complications [4].

Oat (Avena sativa) favorably affects cardiovascular system, because it lowers cholesterol and regulates blood pressure thanks to high content of flavonoids and antioxidants, it strengthens the immune system and improves a complete blood count, stabilizes blood sugar level, and therefore it is important in nutrition of persons with type 2 diabetes. Daily use of oat flakes ( $30 \mathrm{~g}$ ) meet requirements of daily chromium needs ( $150 \mathrm{~g}$ ), which is anyway deficient in daily nutrition due to high refinement of food, and amounts only $30 \mathrm{~g}$. Oat products and oat flakes in nutrition are important for preventive reasons with healthy persons and for normalization with persons who have high fat levels and/or diabetes [5].

Jerusalem artichoke (Helianthus tuberosis) affects blood sugar level favorably. Its most valuable component is inulin. Dry grinded Jerusalem artichoke contains $70 \%$ inulin. It is important to underline that fructose is a result of inulin hydrolyze, which is an excellent replacement for glucose. It improves metabolism: lowers cholesterol and triglycerides in blood which prevents further development of atherosclerosis and cardiovascular complications. It improves microcirculation, blood vessels condition and thereby reduces the seriousness of diabetes complications. It strengthens immune system and reduces proneness to infection. It improves liver condition and gastrointestinal tract, it has probiotic effect etc. It affects favorably reducing of body weight with obese people. It contains important nutritive components and healing properties and it is therefore widely used. By cutting and drying (dehydration of 4-6\% moist), grinding and packing of the powder thus obtained, long-term preservation of inulin, minerals and main biologically active substances is achieved [6].

Cinnamon (Cinnamomum verum, Cinnamonum zeylanicum) is recognized as a cure for obesity, glucose intolerance, diabetes mellitus and dyslipidemia. Integrative medicine is a new concept that combines conventional treatment with evidence that is based on complementary therapy. Cinnamon has a potential to be useful addition to therapy at patients with type 2 diabetes, and in aim to provide more clear evidence, long-term studies whose goal is to determine the efficiency and safety of cinnamon are required. It appears that unlike Cinnamomum cassia that has high contents of coumarin, Cinnamom verum with low coumarin contents is safer for use [7].

\section{Biologically Active Substances: TopiGluk's Healing Basis}

TopiGluk can be rated among "functional food", because beside nutritive effects, its biological properties positively affect health and other body functions. It is a fact that TopiGluk contains many different bioactive compounds and micro elements that human organism needs. TopGluk contains numerous nutritive and nonnutritive nutraceutics.

TopiGluk contains a unique carbohydrate complex of fructose and its polymers, fructo oligosaccharides and inulin [8]. Inulin is the only natural polysaccharide that is composed of $95 \%$ fructose. Inulin is not absorbed in stomach, one-part splits with the help of carbohydrate acid into short fragments composed of fructose and individual fructose molecules that penetrate into blood circulation. The remaining inulin is quickly eliminated after binding great number of unnecessary body substances, such as heavy metals, radionuclides, cholesterol crystals, fatty acids and various toxic substances that have damaging effect and that come from food and intestinal microbial activities. Apart from that, inulin significantly stimulates contractility of intestine, which speeds up organism purifying from toxins, non-digested food and dangerous materials. This is significantly helped by dietary fibers presence.

Inulin has visible probiotic effect because it contributes to establishment of normal intestinal microflora. Fructose originates from inulin. It is a dietary sugar that is able to participate in the same metabolic processes like glucose, by replacing it in case of an absolute or relative insulin deficiency. 


\section{Current Research in Diabetes \& Obesity Journal}

TopiGluk contains minerals that have different functions in organism. It is particularly visible in content of iron, silicon, potassium, magnesium, chromium and zinc. Chromium, magnesium and zinc are important for diabetes. Chromium reduces postprandial glucose increase in blood, improves glucose tolerance, reduces level of required insulin - it is recommendable with obese diabetics because it lowers level of cholesterol and triglycerides in blood. Magnesium enables optimal function of neurons and improves production of insulin in type 2 diabetes. Zinc is involved in synthesis, secretion and insulin usage, it has a preventive role in preventing degeneration of $\beta$-cells of pancreas, and zinc deficit is related with slow sore healing as a common problem with diabetics.

\section{Importance of TopiGluk use from the Aspect of Glucose Homeostasis}

It is expected that TopiGluk use in type 1 diabetes affects favorably glycemia level. Importance of TopiGluk use from the aspect of glucose homeostasis is the most visible in prevention and therapy of type 2 diabetes. It is achieved in the following manner: by reducing glucose absorption from intestine to blood; increasing glucose utilization; increasing cell sensitivity to insulin; reducing insulin resistance; increasing endogenous insulin production; increasing glycogen in liver. Indirect impact on preventing atherosclerosis and vascular complications of diabetes through reduction of cholesterol and triglycerides is also important.

Long-term impact on reduction of body weight with obese patients is particularly important, because obesity is one of the most significant risk factors for diabetes occurrence that can be affected. So, there is a favorable impact on liver condition and functioning of gastrointestinal tract, especially in the context of probiotic impact and gastroenteropathy as a frequent complication of diabetes. All the aforementioned leads to a better health, efficiency increase and improvement of quality of life of patients with diabetes.

Persons with diabetes, particularly with unsatisfactory glycoregulation, often have micronutrients deficiency. Hyperglycemia causes increased diuresis that aims to extract extra sugar from the organism, but increased excretion of vitamins and minerals happens at that time as well. Many diabetics are obese and they apply reduction diets that can often be deficient, so the release of free radicals is increased. Many minerals and vitamins that help in diabetes are represented in TopiGluk.

\section{Other Aspects of TopiGluk Use}

TopiGluk strengthens immune system, it helps elimination of toxins, radionuclides and heavy metals from the body, which is important for inhabitants of urban environments, where pollution levels are very high.
There is a favorable impact on normalization of lipid profile and indirectly on atherosclerosis, hyper tension and coronary disease.

It is recommended with obese persons, it affects favorably liver and gastrointestinal tract, helps with obstipation and in regulation of intestinal microflora.

\section{TopiGluk as a Supplement in Bread Manufacturing}

TopiGluk use as a supplement to base mixture for bread production can reduce starch presence, which- by digestionreleases glucose and lowers energy value of the product. In production procedure, use of TopiGluk affects more intensive baking of the dough; it improves the structure and elasticity of crumbs, increases percentage of moisture binding and prevents the bread to stiffen quickly. TopiGluk affects physical and organoleptic properties of the baked bread; it affects porosity, compressibility and bread volume. This bread has a special smell and taste. Adding TopiGluk affects favorably the expiry date, which is probably the consequence of impact of ingredients that have antimicrobial effect.

TopiGluk bread was developed for the nutrition of patients with type 2 diabetes. The bread was developed as a private brand of the Delhaize Serbia distributor, in cooperation with partners for product development - international company Puratos and national bakery products manufacturer Alimpije, and with a partner for product improvement ZZ Zdravlje . It is produced with a commercial name Glukofit. This bread has increased contents of proteins, fats, organic and mineral elements, vitamins, which enables it to be recommended for therapeutic and preventive nutrition.

\section{Significance of TopiGluk use as an Ingredient of a}

\section{Bread Mixture}

Analysis of glycaemia on a random sample of patients after consuming three types of bread was done: rye bread (blue line), bread made of base mixture without adding TopiGluk (green line) and bread that contains TopiGluk (red line). Graphic chart shows the average glycaemia jump, after consuming the same amount of different bread, at every point of time comparing to the initial base glycaemia value for each bread type. The Chart 1 shows that the smallest postprandial glycaemia jump is after consuming bread that contains TopiGluk.

\section{Conclusion}

TopiGluk that contains biologically active principles of herbal origin has a significant benefit in nutrition form the aspect of glucose homeostasis preservation. Bread that contains TopiGluk is recommended at population with reduced glucose tolerance and with increased risk for diabetes occurrence, firstly because 


\section{Current Research in Diabetes \& Obesity Journal}

of the benefit to glucose homeostasis. With the manifest diabetes existence, use of TopiGluk as a supplement to the mixture for bread is very important, because it reduces postprandial glycaemia jump. TopiGluk as an ingredient of the mixture for a special bread type is very important in nutrition from the aspect of glucose homeostasis.

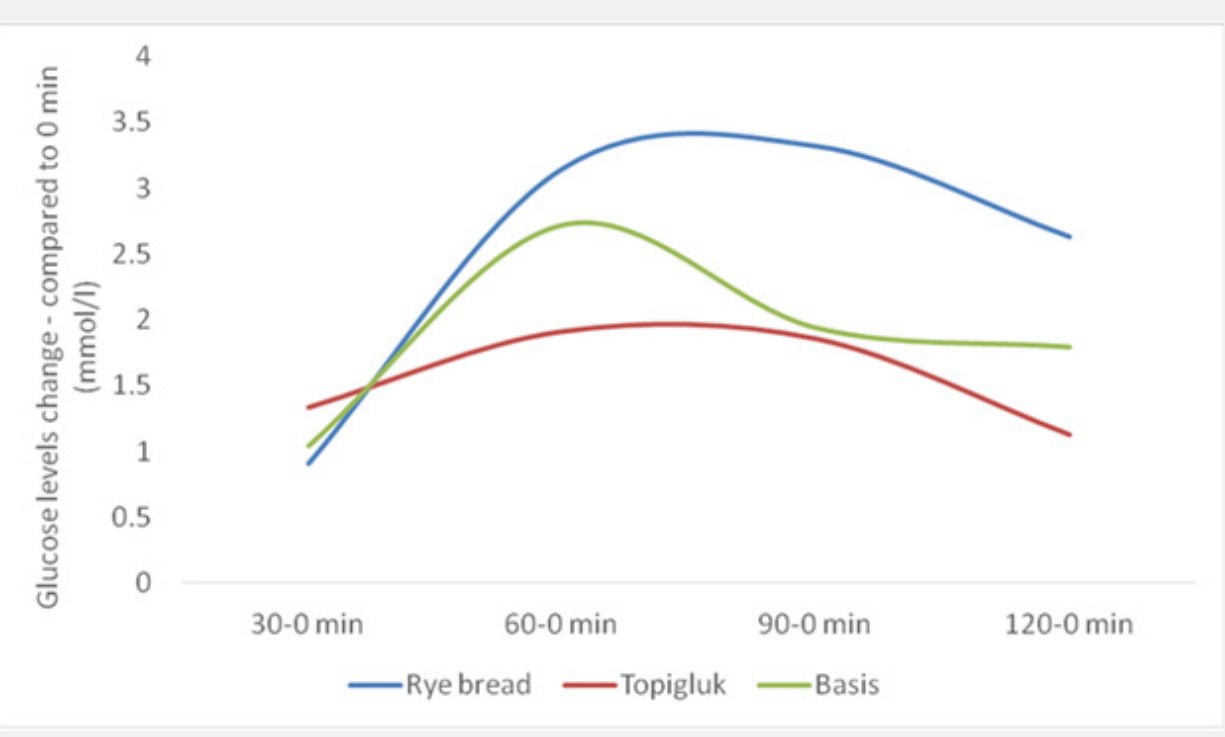

Chart 1: Change of glycaemia value related to the initial value (0 minute).

\section{References}

1. Chinelo Ezeani, Ifeoma Ezenyi, Theophine Okoye, Charles Okoli (2017) Ocimum basilicum extract exhibits antidiabetic effects via inhibition of hepatic glucose mobilization and carbohydrate metabolizing enzymes. J Intercult Ethnopharmacol 6(1): 22-28.

2. Ashraf R, Khan RA, Ashraf I (2011) Garlic (Allium sativum) supplementation with standard antidiabetic agent provides better diabetic control in type 2 diabetes patients. Pak J Pharm Sci 24(4): 565-570.

3. N Neelakantan, M Narayanan, Russell J de Souza, Rob M van Dam (2014) Effect of fenugreek (Trigonella foenum - graecum L.) intake on glycemia: a meta-analysis of clinical trials. Nutr J 13: 7.

4. Akash MS, Rehman K, Tariq M, Chen S (2015) Zingiber officinale and Type 2 Diabetes Mellitus: Evidence from Experimental Studies. Crit Rev Eukaryot Gene Expr 25(2): 91-112.

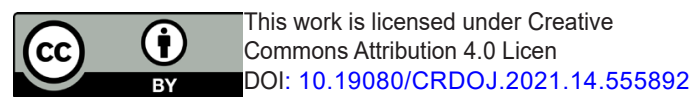

5. Qingtao Hou, Yun Li, Ling Li, Gaiping Cheng, Xin Sun, et al. (2015) The Metabolic Effects of Oats Intake in Patients with Type 2 Diabetes: A Systematic Review and Meta-Analysis, Nutrients. 7(12): 10369-10387.

6. Chang WC, Jia H, Aw W, Saito K, Hasegawa S, et al. (2014) Beneficial effects of soluble dietary Jerusalem artichoke (Helianthus tuberosus) in the prevention of the onset of type 2 diabetes and non-alcoholic fatty liver disease in high-fructose diet-fed rats. Br J Nutr 112(5): 709-717.

7. Arjuna B Medagama (2015) The glycaemic outcomes of Cinnamon, a review of the experimental evidence and clinical trials. Nutr J 14: 108.

8. Wassef Girgiss Nicola, Aly Mohamed Ezz El-Arab, Mina Wassef Girgiss, Dawoud Fakhry Habib, Nadia A Mohamed (2015) Is there a role of inulin in the management of type 2 diabetes mellitus? International Journal of Pharm Tech Research 8(10): 01-09.

\begin{tabular}{|l|}
\hline \multicolumn{1}{|c|}{ Your next submission with Juniper Publishers } \\
will reach you the below assets \\
- Quality Editorial service \\
- Swift Peer Review \\
- Reprints availability \\
- E-prints Service \\
- Manuscript Podcast for convenient understanding \\
- Global attainment for your research \\
- Manuscript accessibility in different formats \\
( Pdf, E-pub, Full Text, Audio) \\
- Unceasing customer service \\
Track the below URL for one-step submission \\
https://juniperpublishers.com/online-submission.php
\end{tabular}

